\title{
Anisotropic Absorption and Reflection Spectra of Poly(ethylene terephthalate) Films in Ultraviolet Region
}

\author{
Isuke OUCHI* \\ Plastics Research Laboratories, Teijin Limited, \\ Oyama, Sagamihara, Kanagawa 229, Japan
}

(Received October 15, 1982)

\begin{abstract}
Thin uniaxially drawn poly(ethylene terephthalate) films (down to $0.1 \mu \mathrm{m}$ thickness) were prepared. Their absorption spectra showed clear dichroism having peaks at $300(\perp)$, $243(/ /)$ and $197 \mathrm{~nm}(/ /)$. Analysis of the reflection spectra of thick films by means of the Kramers-Kronig conversion gave absorption spectra almost identical with those obtained from direct absorption measurement. Spectral shapes and possible origins of the vibrational structures were qualitatively discussed. The oscillator strength of each band was obtained on the simple oriented gas model with or without the Lorentz field correction to examine effects of molecular aggregation. PPP calculations with CI were made by use of a model compound, and four ${ }^{1} A_{\mathrm{g}} \rightarrow{ }^{1} B_{\mathrm{u}}$ allowed transitions were predicted to occur at $4.66,5.27,6.33$ and $6.44 \mathrm{eV}$. The latter two transitions were actually observed at $6.2 \mathrm{eV}$. The calculated transition moments suggested the existence of the cis form of carbonyl groups in oriented amorphous phases.

KEY WORDS Poly(ethylene terephthalate) / Ultraviolet Spectra / Anisotropic Spectra / Reflection Spectra / Kramers-Kronig / Oscillator Strength / Lorentz Field / Pariser-Parr-Pople (PPP) / Transition Moment / Vibronic Spectra /
\end{abstract}

Electronic spectra of poly(ethylene terephthalate) (PET) have been occasionally treated in photochemical or photophysical studies of this polymer; absorption spectra were recorded in connection with photodegradation ${ }^{1}$ or photoconduction ${ }^{2,3}$ and luminescence spectra were discussed in terms of intermolecular energy migration. ${ }^{4-9}$ However, few detailed investigations for the assignments and features of absorption bands of PET have been undertaken. For example, anisotropy in the absorption caused by orientation of the molecules has been reported only in our preliminary note ${ }^{10}$ and, in part, by Padhye et al. ${ }^{11}$

Usually, PET films are transparent in the visible and near ultraviolet regions with a sharp rise in absorption at about $310 \mathrm{~nm}$. Therefore, the entire absorption spectrum may be obtained only with the use of films as thin as about $1 \mu \mathrm{m}$ or less. In this work, such thin films of PET were prepared and their absorption spectra were measured.
Moreover, polarized reflection spectra of PET films were taken and the absorption coefficients and other optical constants in stretching and transverse directions were calculated using the KramersKronig conversion formula. Analysis of reflection spectra by the Kramers-Kronig relationship has been made for a number of inorganic and organic crystals. This means has also been applied to polymers: polyethylene ${ }^{12}$ and poly(tetrafluoroethylene $)^{13}$ in the infrared region and polystyrene ${ }^{14}$ in the vacuum ultraviolet and sulfur nitride polymers, ${ }^{15,16}$ poly(ethylene 2,6-naphthalate) ${ }^{17}$ and polyimide ${ }^{18}$ in the ultraviolet region. However, at present, this does not seem to be a standard technique in polymer characterization. In this paper, it is shown that the polarized reflection spectroscopy can be applied to oriented thick films, whose higher absorbance prevents absorption measurement at short wavelengths, and that it gives basically the same absorption spectra as obtained from

\footnotetext{
* Present address: Central Research Laboratories, Teijin Limited, Asahigaoka, Hino 191, Tokyo.
} 
direct absorption measurement.

Furthermore, from the anisotropic absorption and reflection spectra, the oscillator strength of each absorption band was calculated using the oriented gas model with or without the Lorentz field correction to examine the effects of molecular aggregation. Also, vibronic structures are briefly discussed in comparison with those of poly(ethylene isophthalate) (PEI) and its low molecular weight analogues.

In PET, the $\pi$ electron systems are, in the first approximation, independent of neighbouring repeat units because of the existence of intervening $-\mathrm{CH}_{2} \mathrm{CH}_{2}-$ groups. Hence, the residue

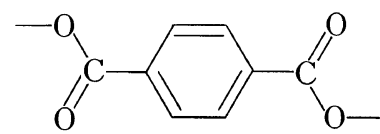

may be regarded as a model compound for PET. For this model, semi-empirical molecular orbital calculations based on the Pariser-Parr-Pople (PPP) approximation $^{19}$ were made to obtain the wavefunctions, transition energies and polarization of the transition moments. A few comments are made on the conformation of PET from the calculated results.

\section{EXPERIMENTAL}

\section{Preparation of Thin Films}

Three methods were used. In the first, the PET melt extruded through a slit of a flat die was allowed to fall by gravity. The screw of the extruder was then stopped so as to make the flat melt fall slowly. An amorphous thin film was quickly collected by hands. It was then drawn uniaxially by a batchwise stretcher provided with metal clamps to hold the four edges of the film. In this manner, a uniaxially oriented film as thin as $1 \mu \mathrm{m}$ was obtained after drawn four to five times.

In the second method, a melt was blown at one end of a glass tube into a baloon shape. This method was applied to PEI.

In the third method, ${ }^{20}$ a $3 \%$ o-chlorophenol solution of PET was made to stand overnight and then cast onto a microscope glass plate which had been made clean with a detergent, water and acetone. Following immersion in acetone for $30 \mathrm{~min}$, the glass plate was dried in air, and then pushed down vertically into the $o$-chlorophenol solution very slowly. After $20 \mathrm{~min}$, the plate was drawn slowly and dried in air. The dried film was scratched along the edge with a tip of tweezers, quickly put into water and taken out. Then, it was slowly immersed in water obliquely to peel it off the plate. With extreme care, the film was fixed between two supporting frames made of thick PET films of $100 \mu \mathrm{m}$ or thicker, dried, and stretched by a tensile tester at a rate of $50 \% / \mathrm{min}$. In this way, PET films as thin as $0.1 \mu \mathrm{m}$ were prepared.

\section{Measurement of Thickness}

Film thickness was measured by several methods. A digital electronic micrometer was used for relatively thick films. For films of about $1 \mu \mathrm{m}$, the interference phenomenon was used. In the interferent part of the absorption curve, wavelengths corresponding to maximum $\left(\lambda_{\max }\right)$ and minimum $\left(\lambda_{\text {min }}\right)$ transmissions were found and the film thickness $d$ was calculated from

$$
d=\frac{\left(2 m^{\prime}+1\right) \lambda_{\min }}{4 n} \text { or } \quad d=\frac{m^{\prime} \lambda_{\max }}{2 n}
$$

where $n$ is the refractive index and $m^{\prime}$ is an integer. $^{21}$

For extremely thin films, part of the film was cut out while still on the glass plate and placed under a profilometer. The distance between the top surface of the film and the surface of the glass plate was measured by having a needle contact with both surfaces. The thickness of a drawn film was estimated by dividing the thickness of the cast film by the draw ratio.

\section{Measurements of Absorption Spectra}

A Shimadzu MPS-5000 UV spectrometer was used to carry out absorption measurements. Nitrogen was continuously made to flow through the spectrometer for measurements in the shorter wavelength region. For polarized light, "Polacoat" polarizers ${ }^{22}$ were placed in front of both the sample and the reference.

For measurements at the liquid nitrogen temperature, a metal Dewar with quartz windows was designed, in which a sample film was held between thick copper frames and cooled indirectly by liquid nitrogen.

\section{Measurements of Reflection Spectra}

The same spectrometer mentioned above was 


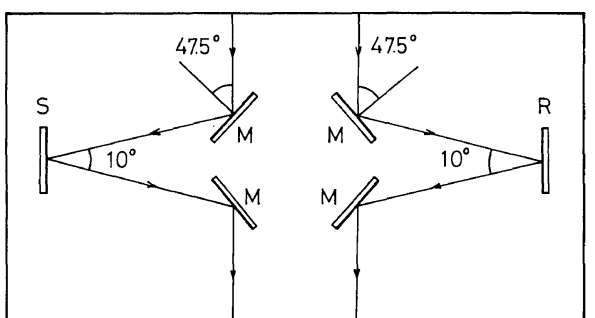

Figure 1. Schematic drawing of the attachment for reflection measurement. $\mathbf{M}$, quartz plate; $\mathrm{R}$, aluminum vaporized plate as reference reflector; $\mathrm{S}$, specimen.

used with an attachement, schematically illustrated in Figure 1, for reflection measurement. Each incident beam onto a quartz plate at an angle of $47.5^{\circ}$ was reflected at the surface with a very small component of the electric vector in the plane of incidence. The beam was incident either on the sample film or on the reference plate at an angle of $5^{\circ}$. The reflected light was then incident on the second quartz plate at an angle of $47.5^{\circ}$ and admitted into the photomultipliers. The angle $47.5^{\circ}$, instead of the Brewster angle of quartz $57^{\circ}$, was selected to make the whole arrangement much simpler. By so doing, mixing of the components of the electric vector in the plane of the paper was estimated to be less than $4 \%$. The deflection of $5^{\circ}$ from the normal incidence caused an error corresponding to $3 \%$ deviation of the refractive index of the specimen. A quartz plate coated with evaporated aluminum was used as the reference plate.

In the transparent and semi-transparent regions, the intensity of reflected light should be corrected for multiple beam reflection. When the film is much thicker than the wavelength, the intensity of reflected light $I^{(\mathrm{r})}$ is expressed in terms of the intensity of incident light $I^{(\mathrm{i})}$ and the reflectivity $R$ as

$$
I^{(r)}=\frac{2 R}{(1-R)^{2}} I^{(i)} \quad(1) \text { in the transparent region }
$$

and

$$
I^{(r)}=\frac{\left(1+t^{4}\right) R}{\left(1-R t^{2}\right)^{2}} I^{(i)}
$$

(2) in the semi-transparent region, where $t$ denotes the transmission of light for one traverse across the film. ${ }^{23}$ From eq 1 and 2, the reflectivity $R$ can be evaluated.

Actual measurements were made in the range $190-740 \mathrm{~nm}$. The apparent reflectivity was read at
$1 \mathrm{~nm}$ intervals for $190-370 \mathrm{~nm}$ and at $10 \mathrm{~nm}$ intervals for $370-740 \mathrm{~nm}$.

\section{Calculation Methods}

Calculations in the Kramers-Kronig analysis and for the lattice sum of dipole fields were made by means of micro-computers (Sord M-200 and Oki If800 Model 20). Semi-empirical molecular orbital calculations using the Hückel and PPP methods were carried out by an IBM 370/168 computer. Computer programs were taken from Kikuchi's book, ${ }^{24}$ with some modifications and additions made.

\section{RESULTS AND DISCUSSION}

\section{Absorption Spectra of Solution}

As low molecular weight analogues of PET, dimethyl terephthalate (DMT) and bishydroxyethyl terephthalate (BHET) were used. These compounds were solved in methanol, trifluoroacetic acid, dioxane or chloroform. Water was also used as the solvent for BHET.

The absorption spectra of DMT in methanol are shown in Figure 2a. The first absorption band, or the lowest energy band, reveals a vibronic structure having maxima at $286 \mathrm{~nm}$ and $294 \mathrm{~nm}$, with a molar extinction coefficient $(\varepsilon)$ of $1.7_{3} \times 10^{3} \quad\left(10^{3}\right.$ $\mathrm{cm}^{2} \mathrm{~mol}^{-1}$ ). The second absorption band has a maximum at $241 \mathrm{~nm}$, with $\varepsilon$ of $1.8_{7} \times 10^{4}$. The oscillator strength $(f)$ was calculated from ${ }^{25}$

$$
f=\frac{10^{3} \ln 10 \cdot m c^{2}}{\mathrm{e}^{2} N_{\mathrm{A}}} \int \varepsilon(\sigma) \mathrm{d} \sigma=4.32 \times 10^{-9} \int \varepsilon(\sigma) \mathrm{d} \sigma
$$

where $m$ is the mass of an electron, $c$ the light velocity, $N_{\mathrm{A}}$ Avogadro's constant, and $\sigma$ the wave number. The calculation gave $f=0.009_{2}$ and 0.34 for the first and second absorption bands, respectively.

The absorption spectra of BEHT has almost the same shape as those of DMT, as shown in Figure $2 b$.

The peak wavelength of the first band varied with the solvent; $292 \mathrm{~nm}$ in $\mathrm{CF}_{3} \mathrm{COOH}, 290 \mathrm{~nm}$ in $\mathrm{H}_{2} \mathrm{O}$, $288 \mathrm{~nm}$ in $\mathrm{CHCl}_{3}, 286 \mathrm{~nm}$ in dioxane and in $\mathrm{CH}_{3} \mathrm{OH}$. Thus, the peak moved toward longer wavelength as the solvent polarity increased. The peak at $295 \mathrm{~nm}$ shifted in a similar manner but was smeared in $\mathrm{CF}_{3} \mathrm{COOH}$ and in $\mathrm{H}_{2} \mathrm{O}$. The red shift 


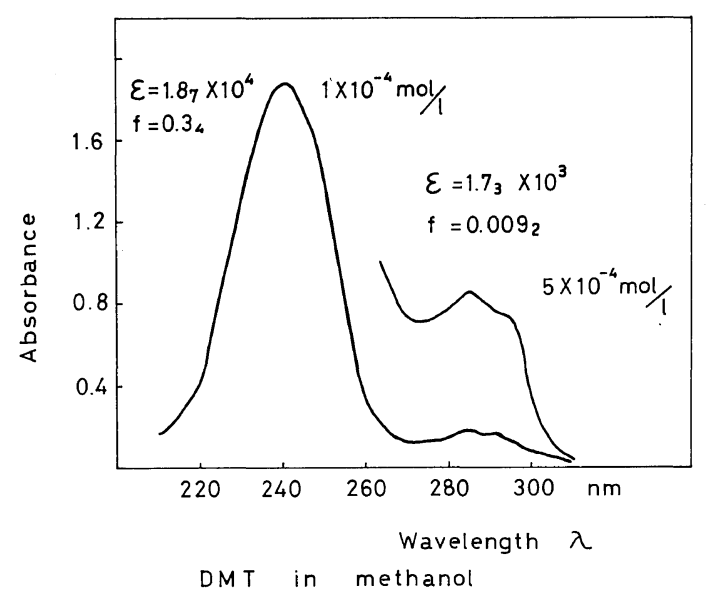

(a)

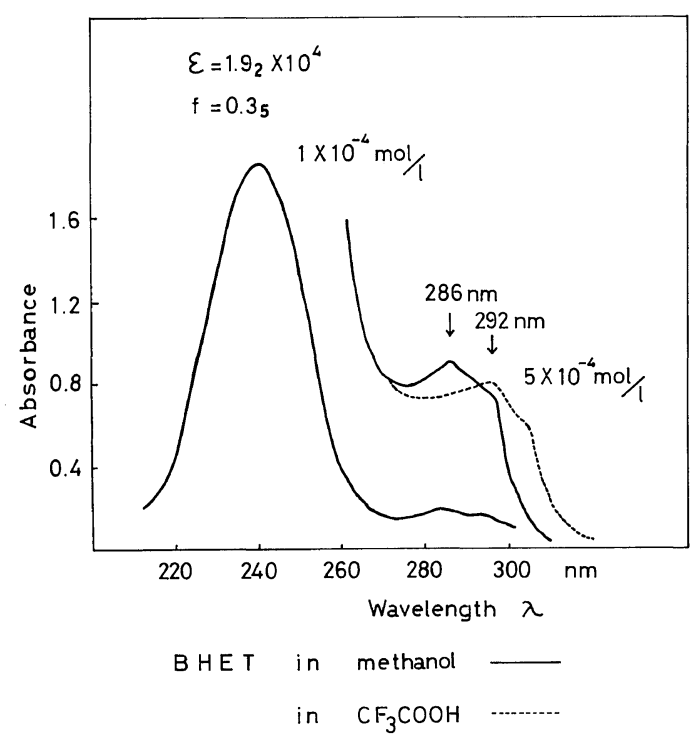

(b)

Figure 2. a. Absorption spectra of DMT solution in methanol. b. Absorption spectra of BHET solution in methanol (-) and in trifluoroacetic acid (------).

phenomenon was also observed in DMT.

Generally, compounds with carbonyl groups such as acetophenone slightly absorb light at about $280-320 \mathrm{~nm}$. This corresponds to the $n \rightarrow \pi^{*}$ transition, where a nonbonded electron of the oxygen atom is excited to the $\pi^{*}$ orbital. In the $n \rightarrow \pi^{*}$ transition, molar extinction coefficients are generally of the order of 10 and absorption bands usually appear at shorter wavelengths when the

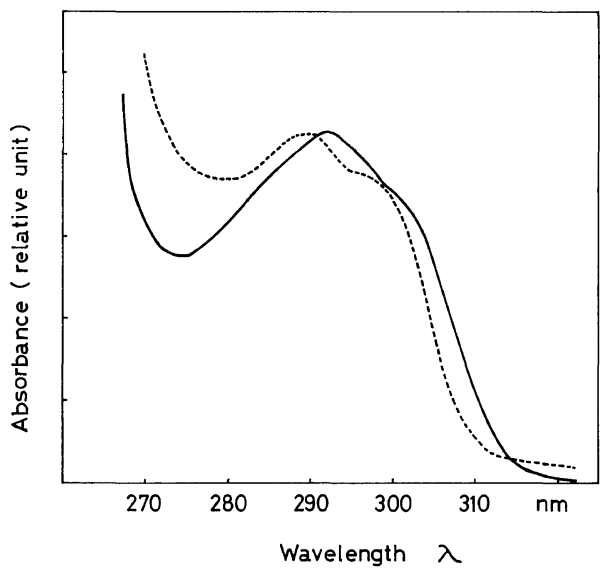

Figure 3. Absorption spectra of PET in trifluoroacetic aicd (-) and as amorphous film (-----).

solvent is more polar. ${ }^{26}$ Although DMT and BHET have carbonyl groups, the behavior of their first absorption bands was completely different from that of $n \rightarrow \pi^{*}$, as described above. Therefore, the first absorption band must be associated with the $\pi \rightarrow \pi^{*}$ transition. According to Nurmukhametov et al. ${ }^{27}$ the $n \rightarrow \pi^{*}$ band is hidden under the first absorption band in the case of terephthalic acid.

The absorption spectrum of PET in $\mathrm{CF}_{3} \mathrm{COOH}$ is very similar to that of BHET in the same solvent, as shown in Figure 3. Also, the solution spectra of PET in hexafluoro-2-propanol reported by Cheung et al. ${ }^{8}$ revealed bands at 291,245 and $191 \mathrm{~nm}$. So far, a possible $n \rightarrow \pi^{*}$ band has not been found in any of PET solution spectra.

\section{Polarized Absorption Spectra}

Polarized absorption spectra of a PET film drawn three times uniaxially to $120 \mathrm{~nm}$ thick are shown in Figure 4, which clearly shows dichroism. The first absorption band absorbs more light with the electric vector perpendicular to the stretching direction and in the plane of the film $(\perp)$ than that with the electric vector parallel to the stretching direction $(/ /)$. The second absorption band absorbs more parallel polarized light than perpendicularly polarized light. The third absorption band at $197 \mathrm{~nm}$ absorbs slightly more parallel polarized light than perpendicularly polarized light.

The spectral properties are summarized in Table I, which includes the oscillator strengths calculated by eq 3 with no correction for band overlapping. 
The dichroism of PET absorption spectra was first pointed out in our preliminary report. ${ }^{10}$ Later, Padhye et al. ${ }^{11}$ also observed anisotropy in the first absorption band. Recently, anisotropic absorption in polyethylene in the vacuum ultraviolet region was reported by Hashimoto et al. ${ }^{28}$ The anisotropy of light absorption is common to linear polymers in any wavelength region.

The first and second absorption bands at the wavelengths listed in Table I mostly agree with the data of Marcotte et al. ${ }^{1}$ and of Takai et al. ${ }^{2,3}$ The third absorption band corresponds to that of the solution spectrum reported by Cheung et al. ${ }^{8}$ and also to those of DMT.

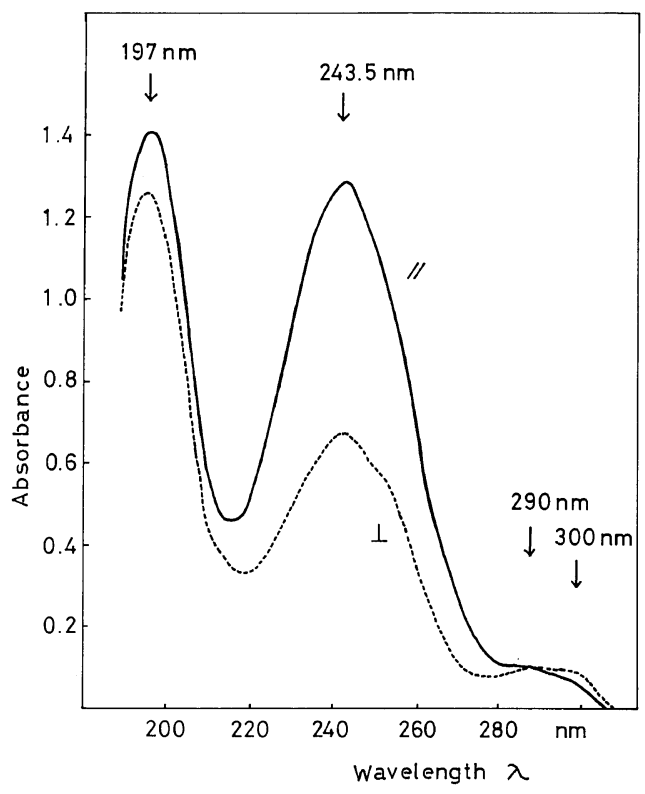

Figure 4. Polarized absorption spectra of a three times uniaxially drawn PET film 120 nm thick.
To examine the first absorption band in more detail, films of about $1 \mu \mathrm{m}$ thick were necessary. Low temperature $(169 \mathrm{~K})$ spectra of such films drawn four times are shown in Figure 5. Two peaks corresponding to vibrational structures are evident and the third peak at $279 \mathrm{~nm}$ is discernible in the low temperature spectra. For the second vibrational band $\left(A^{1}\right)$ located at about $290 \mathrm{~nm}$, there is a difference in peak position between parallel and perpendicular bands.

The wavenumber difference between the first $\left(A^{0}\right)$ and second $\left(A^{1}\right)$ vibrational components is plotted against the draw ratio in Figure 6. The corresponding energy difference does not change greatly in the perpendicular bands, but increases with the draw

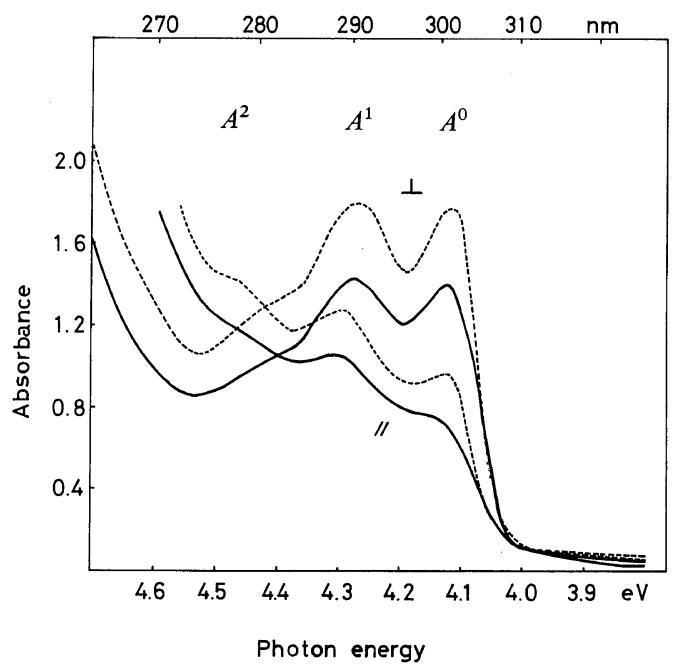

Figure 5. Polarized absorption spectra in the first absorption region of a four times uniaxially drawn PET film measured at $169 \mathrm{~K}$ : - - , non heat treated; ----, heat treated at $200^{\circ} \mathrm{C}$ for $10 \mathrm{~min}$.

Table I. Spectral properties of a uniaxially drawn $(\times 3)$ PET film $120 \mathrm{~nm}$ thick Oscillator strengths are estimated from eq 3 .

\begin{tabular}{|c|c|c|c|c|c|c|}
\hline \multirow{2}{*}{ Polarity } & \multicolumn{2}{|c|}{ 1st abs. band } & \multicolumn{2}{|c|}{ 2nd abs. band } & \multicolumn{2}{|c|}{ 3rd abs. band } \\
\hline & /I & $\perp$ & /I & $\perp$ & // & $\perp$ \\
\hline Abs. maximum $\frac{\left(\lambda_{\max }\right)}{(\mathrm{eV})}$ & \multicolumn{2}{|c|}{$\begin{array}{l}4.13 \\
4.26\end{array}$} & \multicolumn{2}{|c|}{5.09} & \multicolumn{2}{|c|}{6.29} \\
\hline$\varepsilon\left(10^{3} \mathrm{~cm}^{2} \mathrm{~mol}^{-1}\right)$ & $\begin{array}{l}1.2_{2} \times 10^{3} \\
1.6_{2} \times 10^{3}\end{array}$ & $\begin{array}{l}1.4_{2} \times 10^{3} \\
1.6_{2} \times 10^{3}\end{array}$ & $1.5_{3} \times 10^{4}$ & $8.0_{1} \times 10^{3}$ & $1.6_{8} \times 10^{4}$ & $1.4_{9} \times 10^{4}$ \\
\hline $\begin{array}{l}f \\
f_{\|} / f_{\perp}\end{array}$ & \multicolumn{2}{|c|}{$0.8_{5}$} & \multicolumn{2}{|c|}{$1.8_{9}$} & \multicolumn{2}{|c|}{$1.0_{9}$} \\
\hline
\end{tabular}




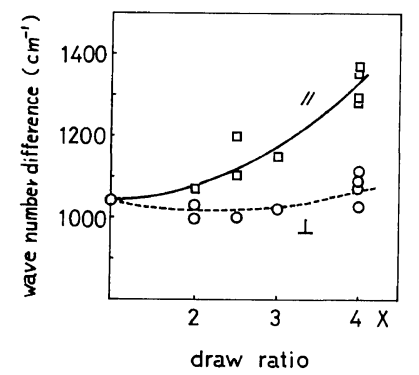

Figure 6. Wavenumber differences of the vibronic bands $A^{0}$ and $A^{1}$ in the first absorption region plotted against draw ratio.

ratio in the parallel bands. One primary reason of this would be that the parallel band of the second absorption band at about $243 \mathrm{~nm}$ is so intense that its tail contributes to the intensity of the first absorption band, significantly at the $A^{1}$ position and somewhat at the $A^{0}$ position. Moreover, the intensities of the parallel and perpendicular bands of the second absorption increase and decrease, respectively, with increasing draw ratio. Therefore, the tail of the former band influences the $A^{1}$ position more strongly with an increase in orientation, while the tail of the latter band less influences the $A^{1}$ position with an increase in draw ratio. If the contribution of the tail of the second absorption band is subtracted, the discrepancy in the peak position of the $A^{1}$ band between parallel and perpendicular spectra decreases considerably (see Table II).

There still remains some difference between the positions of the parallel and perpendicular components of the $A^{1}$ band, as can be seen from Table II. Since each unit cell of the PET crystal contains only one repeat unit, this difference is not due to the Davydov splitting. If the difference is not due to experimental error, it may be explained as follows. In crystalline or non-crystalline solids, there are several modes of intramolecular vibrations which have different energies and various intermolecular interaction intensities, and undergo dissimilar energy changes when excited to an upper electronic state. Overlapping of these vibration adds to complexity and distorts the progression intervals differently in the parallel and perpendicular directions, causing a difference in the peak positions of the bands.

Similar vibrational structures were observed in a
PEI film (Figure 7) and in its low molecular weight analogue, dimethyl isophthalate (DMI). The differences in peak positions between the $A^{0}$ and $A^{1}$ vibronic components of these compounds are compared in Table III.

It should be noticed that the wavenumber differences between the $A^{0}$ and $A^{1}$ components are classified into two groups; DMT, BHET and PET have $A^{0}-A^{1}$ values of about $1100 \mathrm{~cm}^{-1}$, while DMI and PEI have those of about $970 \mathrm{~cm}^{-1}$. This implies that the vibration contributing to the main vibronic structure must originate from the benzene ring vibrations having different frequencies for the $p$ and $m$-substitutes. The first absorption band is due to an allowed electronic transition, as will be described later, so that only totally symmetric modes are concerned with vibronic structures. To the best of our knowledge, no data are available on Raman spectra of PEI or DMI. Therefore, reference is made only to the Raman data of PET. ${ }^{29-31}$ The totally symmetric Raman bands corresponding to the wavenumber differences between $A^{0}$ and $A^{1}$ in Table III would be $1096 \mathrm{~cm}^{-1}$ or the combination of 857 , 1295 and $1615 \mathrm{~cm}^{-1}$. Among them, the band at $1096 \mathrm{~cm}^{-1}$ forms a peak only in the crystalline state so that it is not a main factor causing the vibronic structure. The vibrational structure of the first absorption band of benzoic acid was examined at 296, 213, 138, and $77 \mathrm{~K}^{32}$ Comparison of its temperature dependency with ours prompted us to expect that the temperature $77 \mathrm{~K}$ would increase the resolution considerably in the case of PET as well. Justification for this expectation will require additional research.

Another feature of interest in the first absorption region is the tail of the spectrum. When the absorption of a $160 \mu \mathrm{m}$ amorphous PET film was measured, very weak shoulders were found at $341 \mathrm{~nm}$ and $358 \mathrm{~nm}$, as shown in Figure 8. Their molar extinction coefficients are $\varepsilon=2.9$ and 1.8 , including the background. In consideration of the energy difference between the $A^{0}$ peak and those shoulders, these values of $\varepsilon$ may not be attributed to the transition from vibrational sublevels of the ground state to the lowest excited state. The bands at $341 \mathrm{~nm}$ and $358 \mathrm{~nm}$ were found as fluorescence excitation bands in PET films at room temperature ${ }^{5}$ and in fibers at low temperature. ${ }^{8}$ In the present study, the excitation spectrum revealed a maximum at $343 \mathrm{~nm}$ and three shoulders at $279 \mathrm{~nm}$ and $315 \mathrm{~nm}$ 
Absorption and Reflection Spectra of PET

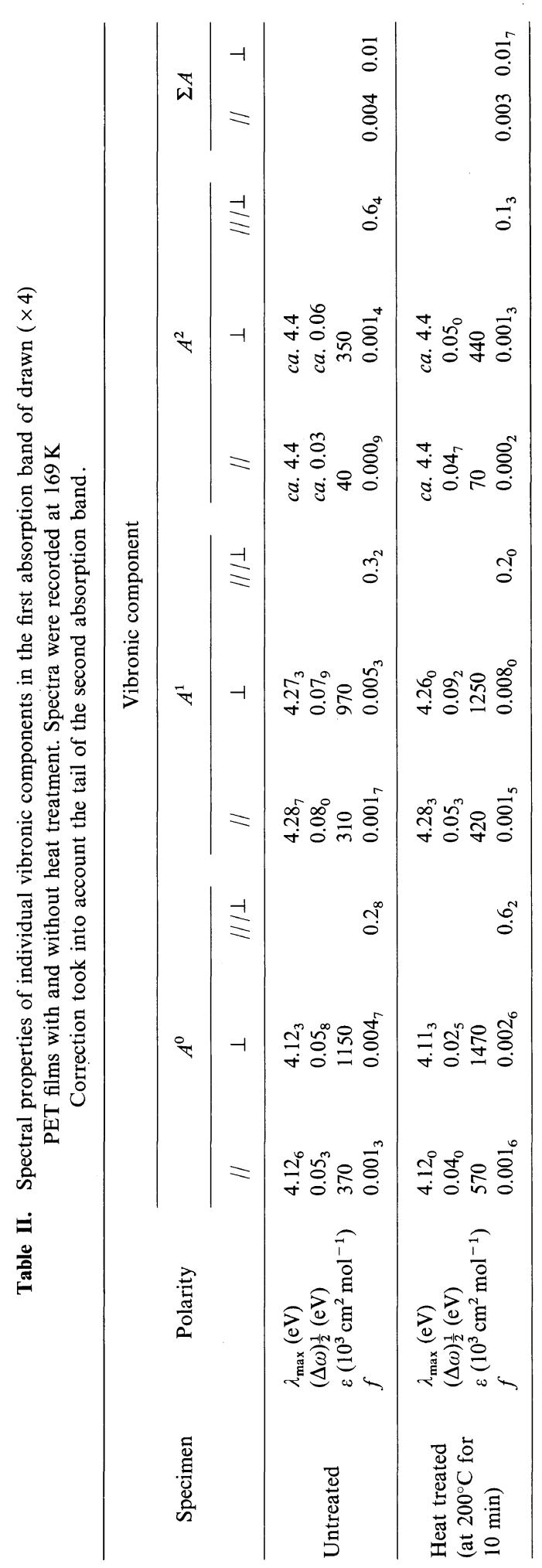




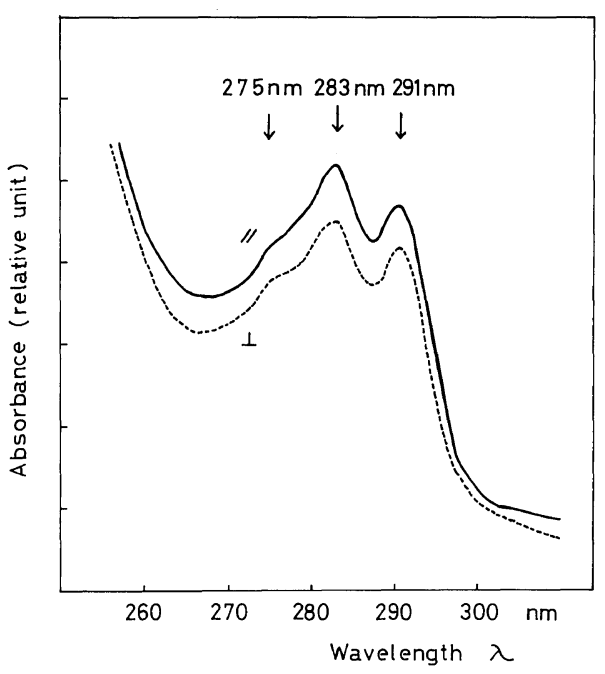

Figure 7. Absorption spectra of a PEI film.

Table III. The vibronic structure of PET, PEI, and their low molecular weight analogues at room temperature

\begin{tabular}{|c|c|c|}
\hline \multirow{2}{*}{ Compound } & \multicolumn{2}{|c|}{ Wave number difference } \\
\hline & $A^{0}-A^{1}$ & $A^{1}-A^{2}$ \\
\hline DMT $\left(\mathrm{CHCl}_{3}\right.$ solution $)$ & $1120 \mathrm{~cm}^{-1}$ & \\
\hline BHET $\left(\mathrm{CHCl}_{3}\right.$ solution $)$ & 1095 & \\
\hline Oligomer $\left(\mathrm{CHCl}_{3}\right.$ solution $)$ & 1195 & 1390 \\
\hline PET (heat treated) $(\perp)$ & 1090 & ca. 1420 \\
\hline DMI ( $\mathrm{CHCl}_{3}$ solution) & 975 & 880 \\
\hline PEI $\left(\mathrm{CHCl}_{3}\right.$ solution $)$ & 975 & ca. 780 \\
\hline PEI (film) & 960 & 860 \\
\hline
\end{tabular}

and possibly at $358 \mathrm{~nm}$, as shown in Figure 9. Therefore, the weak absorption structures at $341 \mathrm{~nm}$ and $358 \mathrm{~nm}$ are linked to the excitation peak at $343 \mathrm{~nm}$ and to the broad shoulder at $358 \mathrm{~nm}$. According to the experiment of Nurmukhametov et al. ${ }^{27}$ the $n \rightarrow \pi^{*}$ band in terephthalic aldehyde exists near $350 \mathrm{~nm}$. Therefore, there is a possibility that the band at $341-343 \mathrm{~nm}$ is due to $n \rightarrow \pi^{*}$. However, since the extinction coefficient is somewhat too small for $n \rightarrow \pi^{*}$, the band could correspond to ${ }^{1} \pi \rightarrow{ }^{3} \pi^{*}$, just as benzene's ${ }^{1} A_{1 \mathrm{~g}} \rightarrow{ }^{3} B_{1 \mathrm{u}}$ appears at $340 \mathrm{~nm}$.

\section{Polarized Reflection Spectra}

The reflection spectra of a five times uniaxially

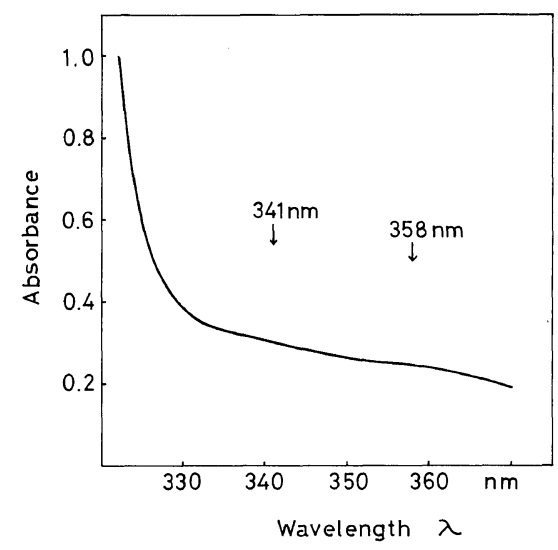

Figure 8. Absorption spectra of an undrawn PET films $160 \mathrm{~nm}$ thick.

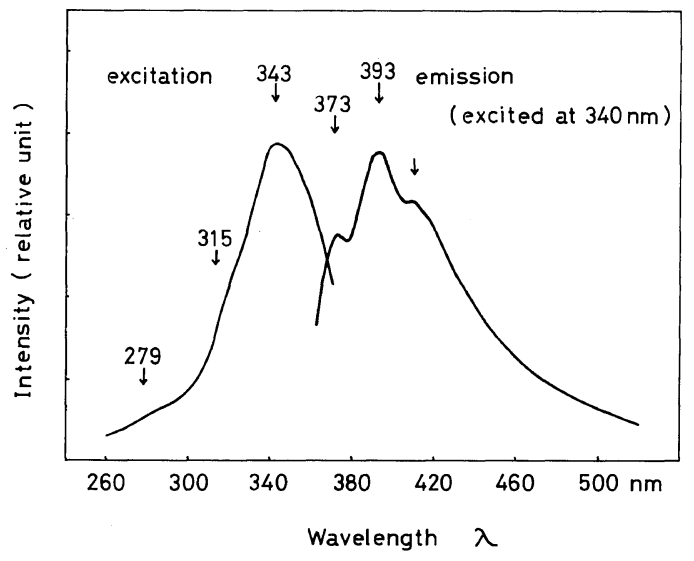

Figure 9. Fluorescence excitation and emission spectra of a PET film at room temperature.

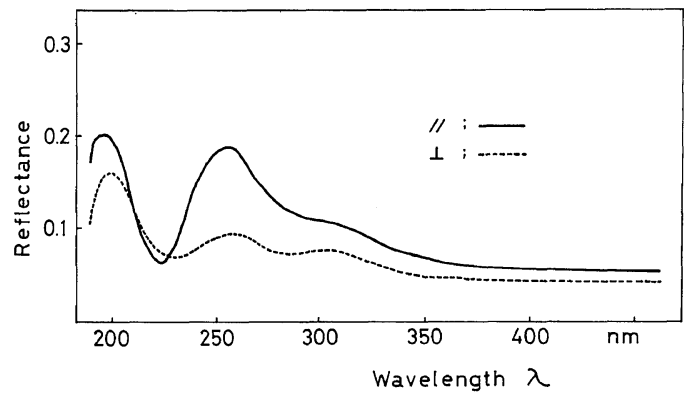

Figure 10. Reflection spectra of a five times uniaxially drawn PET film $47 \mu \mathrm{m}$ thick.

drawn PET film of $47 \mu \mathrm{m}$ thick are presented in Figure 10, where correction for multiple reflection has been made. 
The complex amplitude reflectivity $\hat{r}$ is expressed in terms of the intensity reflectivity $R$ and the phase change $\theta$ of the electric field by reflection ${ }^{33}$ as follows:

$$
\hat{r}(\omega)=\sqrt{R(\omega)} \mathrm{e}^{i \theta(\omega)}
$$

or

$$
\ln \hat{r}(\omega)=\frac{1}{2} \ln R(\omega)+i \theta(\omega)
$$

where $\omega$ is the angular frequency of light. The real and imaginary parts of the complex function $\ln \hat{r}(\omega)$ are not independent of each other and satisfy the Kramers-Kronig relationship, which yields ${ }^{23,34}$

$$
\theta\left(\omega_{i}\right)=\frac{1}{2 \pi} \int_{0}^{\infty} \frac{\mathrm{d}}{\mathrm{d} \omega}(\ln R(\omega)) \ln \left(\frac{\omega+\omega_{i}}{\omega-\omega_{i}}\right) \mathrm{d} \omega
$$

Thus, the phase change $\theta$ at frequency $\omega_{i}$ can be computed provided the reflectivity $R(\omega)$ is measured over the entire range of frequency. In actuality, however, $R(\omega)$ can be measured only over a limited range of $\omega$. Fortunately, owing to the denominator $\left(\omega-\omega_{i}\right)$ in the logarithmic term, if an appropriate approximation is used, the integral may be evaluated with reasonable accuracy from measured $R(\omega)$ over a limited range of $\omega$. In this study, Roessler's approximation ${ }^{35}$ was employed. The results are shown in Figure 11.

The refractive index $n(\omega)$ and the extinction coefficient $\kappa(\omega)$, which are defined by complex refractivity $\hat{n}(\omega)=n(\omega)-i \kappa(\omega)$, are related to $r(\omega)$ and $\theta(\omega)$ through the Fresnel formulae. For normal incidence, we have

$$
\begin{aligned}
& n(\omega)=\frac{1-R(\omega)}{1+R(\omega)-2 \sqrt{R(\omega)} \cos \theta(\omega)} \\
& \kappa(\omega)=\frac{2 \sqrt{R(\omega)} \sin \theta(\omega)}{1+R(\omega)-2 \sqrt{R(\omega)} \cos \theta(\omega)}
\end{aligned}
$$

Figures 12 and 13 illustrate the calculated results for $n$ and $\kappa$. As seen from Figure 12, the calculated values of $n$ at wavelength $585 \mathrm{~nm}$ are $n_{\|}=1.62$ and $n_{\perp}=1.54$, where // signifies the electric vector parallel to the stretching direction $\perp$ the electric vector perpendicular to it but in the plane of the film. On the other hand, the value of $n$ measured with an Abbe refractometer were $n_{\|}=1.66, n_{\perp}=1.58$ and $n_{z}=1.51$ at the same wavelength, where $n_{z}$ is the refractive index when the electric vector is perpendicular to the film plane. Thus, the agreement

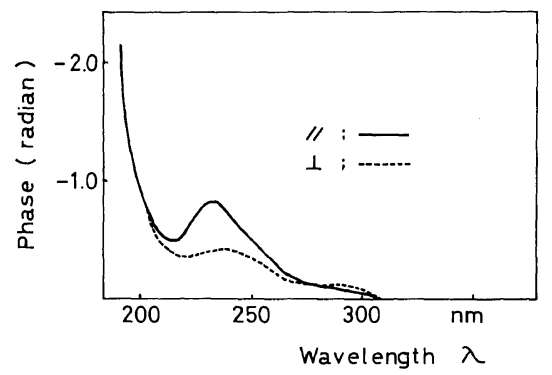

Figure 11. Phase change $\theta$ vs. $\lambda$ as computed from measured reflectivity by the Kramers-Kronig conversion, eq 5. The specimen is the same as that for Figure 10 .

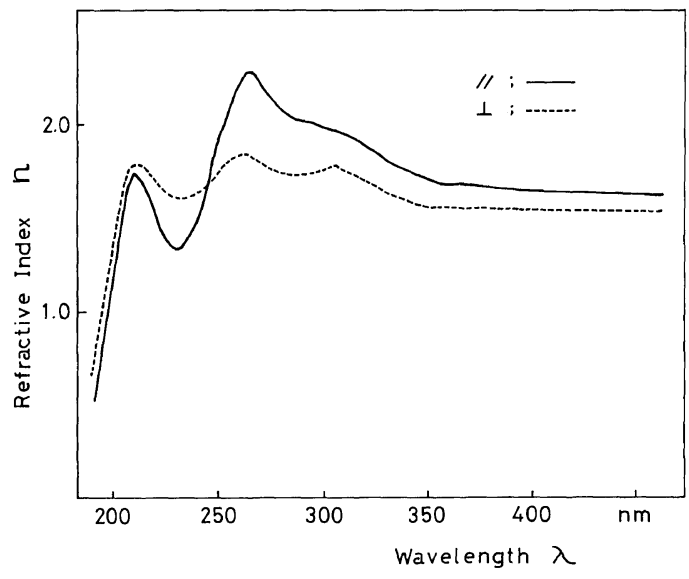

Figure 12. Refractive index $v s$. $\lambda$ as calculated by eq 6 . The specimen is the same as that for Figure 10.

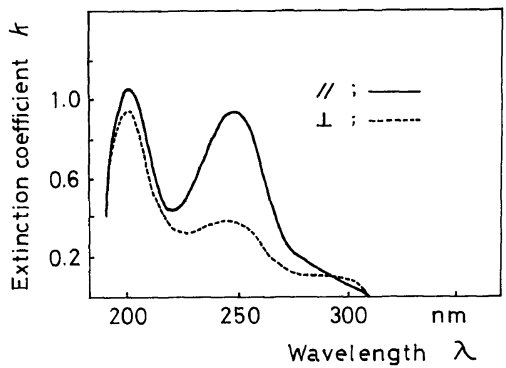

Figure 13. Extinction coefficients $v s$. $\lambda$ as calculated by eq 7. The specimen is the same as that for Figure 10.

between calculated and measured values is fairly satisfactory.

The absorption coefficient $K(\omega)$ is related to $\kappa(\omega)$ by

$$
K(\omega)=\frac{4 \pi}{\lambda} \kappa(\omega)
$$


where $\lambda$ is the wavelength of incident light. The absorption spectra calculated from eq 8 are shown in Figure 14. Their general features are fairly consistent with the absorption spectra of the very thin, three times drawn films shown in Figure 4. The perpendicular band has a higher absorption than the parallel band in the first absorption region, the parallel band is much stronger than the perpendicular band in the second absorption region, and the parallel band is somewhat stronger than the perpendicular band in the third absorption region. The absorption peak positions, along with the reflection peak positions and the molar extinction coefficients, are listed in Table IV. The discrepancies between the absorption peak positions shown in Table I and

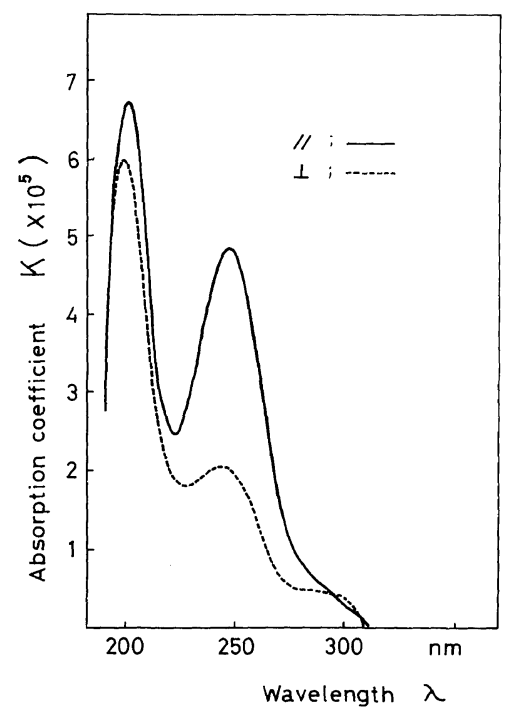

Figure 14. Absorption coefficients $v s$. $\lambda$ as calculated by eq 8 . The specimen is the same as that for Figure 10 .
Table IV are partly due to the samples used (the film for the absorption measurement was very thin and three times drawn, while the film for the reflection measurement was thick and five times drawn) and partly due to the error involved in Roessler's approximation. However, the present results on PET films offer a direct justification of the KramersKronig analysis for the spectra of oriented polymer films in the ultraviolet region. The major advantages of reflection spectroscopy are the use of thick films in shorter wavelengths at which the absorption is intense. In our previous note, ${ }^{17}$ the Kramers-Kronig analysis was applied to poly(ethylene 2,6-naphthalate), for which it was impossible to prepare a film sufficiently thin for absorption measurement.

Oscillator Strength Obtained from Reflection Spectra

The real part $\varepsilon_{1}(\omega)$ and the imaginary part $\varepsilon_{2}(\omega)$ of the complex dielectric constant $\hat{\varepsilon}(\omega), \hat{\varepsilon}(\omega)=$ $\varepsilon_{1}(\omega)-i \varepsilon_{2}(\omega)$, are related to $n(\omega)$ and $\kappa(\omega)$ by

$$
\begin{aligned}
& \varepsilon_{1}(\omega)=n^{2}(\omega)-\kappa^{2}(\omega) \\
& \varepsilon_{2}(\omega)=2 n(\omega) \kappa(\omega)
\end{aligned}
$$

The values of $\varepsilon_{1}$ and $\varepsilon_{2}$ calculated from these equations are shown in Figures $15 \mathrm{a}$ and $15 \mathrm{~b}$. The complex dielectric constant $\hat{\varepsilon}(\omega)$ is related to the complex total polarizability $\hat{\alpha}(\omega)$ by

$$
\begin{aligned}
& \hat{\varepsilon}(\omega)=1+4 \pi \hat{\alpha}(\omega) \\
& \hat{\alpha}(\omega)=N \hat{\alpha}_{0}(\omega)
\end{aligned}
$$

where $N$ is the number of valence electrons per unit volume contributing to optical absorption and $\hat{\alpha}_{0}(\omega)$ is the polarizability per oscillator.

\begin{tabular}{|c|c|c|c|c|c|c|}
\hline \multirow{2}{*}{ Polarity } & \multicolumn{2}{|c|}{ 1st abs. band } & \multicolumn{2}{|c|}{ 2nd abs. band } & \multicolumn{2}{|c|}{ 3rd abs. band } \\
\hline & $/ /$ & $\perp$ & $/ /$ & $\perp$ & $/ /$ & $\perp$ \\
\hline Reflection max. (eV) & & $4.0_{8}$ & $4.8_{2}$ & $4.8_{0}$ & $6.2_{9}$ & $6.2_{3}$ \\
\hline $\begin{array}{l}\text { Calculated } \\
\text { absorption max. }\left(\lambda_{\max }\right)\end{array}$ & $\begin{array}{l}(4.13(\mathrm{sh}) \\
4.27\end{array}$ & $\begin{array}{l}4.13(\mathrm{sh})) \\
4.33\end{array}$ & $5.0_{5}$ & $5.1_{1}$ & $6.1_{8}$ & $6.2_{1}$ \\
\hline$\varepsilon\left(10^{3} \mathrm{~cm}^{2} \mathrm{~mol}^{-1}\right)$ & $\begin{array}{c}\left(3.3 \times 10^{3}\right. \\
1.7_{5} \times 10^{3}\end{array}$ & $\begin{array}{l}\left.3.1_{8} \times 10^{3}\right) \\
2.3_{7} \times 10^{3}\end{array}$ & $3.0 \times 10^{4}$ & $1.29 \times 10^{4}$ & $4.16 \times 10^{4}$ & $3.7 \times 10^{4}$ \\
\hline$\varepsilon_{\|} / \varepsilon_{\perp}$ & \multicolumn{2}{|c|}{$0.7_{4}$} & \multicolumn{2}{|c|}{$2.3_{3}$} & \multicolumn{2}{|c|}{$1.1_{2}$} \\
\hline
\end{tabular}

The classical harmonic oscillator model ${ }^{36}$ and the

Table IV. Spectral properties of a uniaxially drawn $(\times 5)$ PET film $47 \mu \mathrm{m}$ thick by Kramers-Kronig analysis from the reflection spectra 


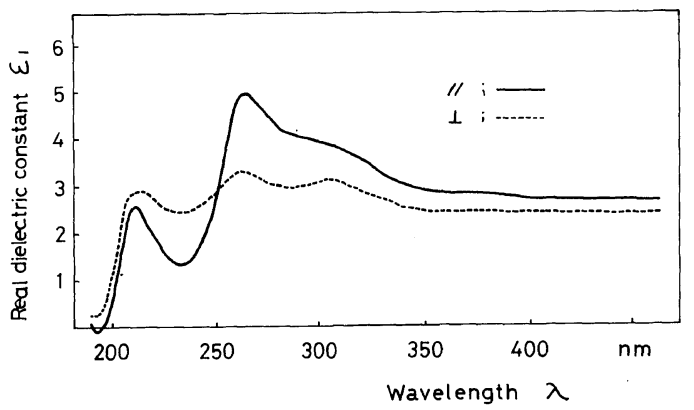

(a)

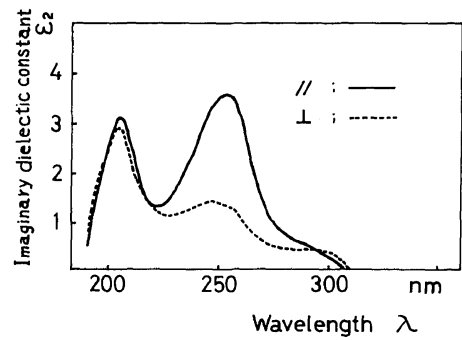

(b)

Figure 15. a. Real part $\varepsilon_{1}(\omega)$ of the complex dielectric constant $\varepsilon(\omega) v s$. $\lambda$. b. Imaginary part $\varepsilon_{2}(\omega)$ of $\varepsilon(\omega) v s$. $\lambda$. The specimen is the same as that for Figure 10.

quantum mechanical treatment ${ }^{37}$ give

$$
\alpha_{0}(\omega)=\frac{e^{2}}{m} \sum_{j} \frac{f_{j}}{\left(\omega_{0 j}^{2}-\omega^{2}\right)+i \gamma_{j} \omega}
$$

where the subscript $j$ distinguishes different oscillators, $\omega_{0 j}$ is the resonance angular frequency, $\gamma_{j}$ is damping constant, and $f_{j}$ is the oscillator strength of the $j$-th mode. From eq 11 and 13, we obtain

$$
\begin{aligned}
\varepsilon_{1}(\omega) & =1+\sum_{j} \frac{f_{j}\left(\omega_{0 j}^{2}-\omega^{2}\right) \frac{4 \pi N \mathrm{e}^{2}}{m}}{\left(\omega_{0 j}^{2}-\omega^{2}\right)^{2}+\omega^{2} \gamma_{j}^{2}} \\
\varepsilon_{2}(\omega) & =\frac{4 \pi N \mathrm{e}^{2}}{m} \sum_{j} \frac{f_{j} \gamma_{j} \omega}{\left(\omega_{0 j}^{2}-\omega^{2}\right)+\omega^{2} \gamma_{j}^{2}}
\end{aligned}
$$

From eq $15^{23}$ it follows that

$$
\frac{4 \pi N \mathrm{e}^{2}}{m} f_{j}=\gamma_{j}\left(\varepsilon_{2}(\omega) \cdot \omega\right)_{\max }
$$

or $^{38}$

$$
f_{j}=\frac{m}{2 \pi^{2} N \mathrm{e}^{2}} \int_{j} \varepsilon_{2}(\omega) \cdot \omega \mathrm{d} \omega
$$

where $\int_{j}$ indicates the integration over the interval

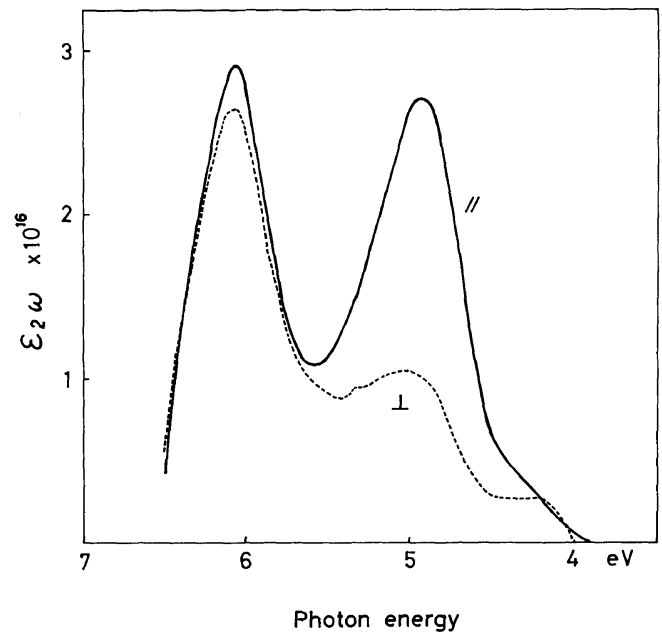

Figure 16. $\varepsilon_{2}(\omega) \cdot \omega v s$. $\omega$. The specimen is the same as that for Figure 10.

Table V. Oscillator strengths in the absorption bands of uniaxially drawn $(\times 5)$ PET films $47 \mu \mathrm{m}$ thick calculated by eq 16

\begin{tabular}{lcccccc}
\hline & $\begin{array}{c}\text { 1st abs. } \\
\text { band }\end{array}$ & \multicolumn{2}{c}{$\begin{array}{c}\text { 2nd abs. } \\
\text { band }\end{array}$} & \multicolumn{2}{c}{$\begin{array}{c}\text { 3rd abs. } \\
\text { band }\end{array}$} \\
\hline \multicolumn{1}{c}{ Polarity } & $\|$ & $\perp$ & $\|$ & $\perp$ & $\|$ & $\perp$ \\
\hline$\left(\varepsilon_{2}(\omega) \cdot \omega\right)_{\max }$ & $4.1-4.2 \mathrm{eV}$ & $4.9_{3}$ & $5.0_{4}$ & $6.0_{6}$ & $6.0_{6}$ \\
$f /$ repeat unit & 0.01 & $0.3_{2}$ & $0.15_{8}$ & $0.2_{9}$ & $0.2_{8}$ \\
$f /$ electron & 0.0007 & $0.02_{2}$ & $0.01_{4}$ & $0.02_{1}$ & $0.02_{1}$ \\
$\gamma(\mathrm{eV})$ & $0.5_{0}$ & $0.7_{1}$ & $0.7_{6}$ & $0.6_{0}$ & $0.6_{4}$ \\
$f_{\|} / f_{\perp}$ & $<1$ & \multicolumn{2}{c}{$2.1_{2}$} & \multicolumn{2}{c}{$1.0_{3}$} \\
\hline
\end{tabular}

associated with the $j$-th resonance absorption. Thus, if $\varepsilon_{2}(\omega) \cdot \omega$ is plotted against $\omega$ as in Figure $16, f_{j}$ is determined with $\gamma_{j}$ which corresponds to the full half-width of each band in the $\varepsilon_{2} \omega$ spectra or by integrating $\varepsilon_{2} \omega$ over the interval described above. The oscillator strength obtained refers to one valence electron. Since there are 14 valence electrons per repeat unit of PET, the oscillator strength per repeat unit can be found by multiplying it by 14 . The results are given in Table V.

The oscillator strength calculated from eq 3 or 16 corresponds to the case where the refractive index is unity. Regardless of the difference in draw ratio and film thicknesses, the oscillator strengths of the PET films described in Tables $I$ and $V$ are fairly close to 
each other. To examine this point, eq 14 and 15 should be treated more rigorously.

When light is incident on an oriented specimen, the electric vector of the incident light induces oscillatory electron motion which, in turn, generates an anisotropic electric field at the sites of other electrons. Let $\boldsymbol{E}_{0}$ be the applied field, and $\boldsymbol{E}_{\text {loc }}$, the local field in the medium. The polarization or dipole moment per unit volume is denoted by $\boldsymbol{P}$, the dipole moment at the $i$-th atom by $\boldsymbol{p}_{i}$, and the displacement vector from the reference point by $\boldsymbol{R}^{\prime}$. Then ${ }^{39}$

$$
\boldsymbol{E}_{\mathrm{loc}}=\boldsymbol{E}_{0}+\frac{4 \pi}{3} \boldsymbol{P}+\sum_{i} \frac{3\left(\boldsymbol{p}_{i} \cdot \boldsymbol{R}_{i}^{\prime}\right) \boldsymbol{R}_{i}^{\prime}-\boldsymbol{p}_{i} \boldsymbol{R}_{i}^{\prime 2}}{\boldsymbol{R}_{i}^{\prime 5}}
$$

The third term on the right hand must be calculated inside a small sphere whose diameter is sufficiently small compared to the wavelength of incident light but large compared to the atomic distance. In this sphere, the phase retardation of the electric vector is negligible. ${ }^{40}$ Therefore, the sum can be evaluated with relative ease. The sum is zero for cubic symmetry systems but not so for other symmetry systems.

Calculation was made on the assumption that all atoms are located at the atomic sites of perfect crystals of PET, with the atomic coordinates of Daubeny et al. ${ }^{41}$ Three cases must be considered: the electric vector $\boldsymbol{E}_{0}$ is parallel to the $x, y$ and $z$ axes of PET crystals. Let the $z$ axis be taken along the $c$ axis of the unit cell of PET, the $y$ axis perpendicular to the $z$ axis and in the plane of the film and the $x$ axis perpendicular to the film plane. Since the unit cell is triclinic, the electric vector parallel to the $y$ axis induces dipole moments not only along the $b$ axis but along the $c$ axis as well. The dipole moment

along the $c$ axis is so small that it was neglected in the present calculations. The value of $p_{i}$ was taken to be proportional to the density of $\pi$ electrons at each atomic site which was calculated by the semiempirical molecular orbital method as described in the following section. For comparison, the case in which all $p_{i}$ 's are equal was also calculated. For the diameter of the sphere, three values were considered: 15,25 , and $50 \AA$. The results are listed in Table VI, which shows that the dipole field at the center of the sphere of radius $50 \AA$ is nearly equal to that of $25 \AA$. Therefore, the radius $50 \AA$ is sufficient for our purpose. This value is reasonable, compared with that calculated by Matsui ${ }^{42}$ for anthrancene. Consideration of electron density did not cause much difference in the results in comparison with the case in which the charge was distributed uniformly at the atomic sites. The following calculation was made on the basis of the former condition.

Since $\boldsymbol{P}$ is the polarization per unit volume

$$
\boldsymbol{P}(\omega)=\sum N_{l} \boldsymbol{p}_{l}(\omega)=\sum N_{l} \alpha_{l}(\omega) \boldsymbol{E}_{\text {loc }}
$$

where $N_{l}$ is the number of the $l$-th dipole $p_{l}$ per unit volume. Since the volume of the unit cell is $218.9 \AA^{3}$, $N=6.394 \times 10^{22}$ and $N_{l}=4.567 \times 10^{21}$.

Define $A$ by

$$
A=\frac{4 \pi}{3}+\frac{1}{N_{0} p} \sum_{i} \frac{3\left(\boldsymbol{p}_{i} \cdot \boldsymbol{R}_{i}^{\prime}\right) \boldsymbol{R}_{i}^{\prime}-\boldsymbol{p}_{i} \boldsymbol{R}^{\prime 2}}{\boldsymbol{R}_{i}^{\prime}}
$$

where $p$ is the average of $p_{i}$. From eq 18 and 19, it follows that

$$
\frac{P}{E_{0}}=\frac{\Sigma N_{l} \alpha_{l}}{1-A \Sigma N_{l} \alpha_{l}}
$$

Table VI. 'Lattice sum of the dipole field $\sum_{i} \frac{3\left(\boldsymbol{p}_{i} \cdot \boldsymbol{R}_{i}^{\prime} \cdot \boldsymbol{R}_{i}^{\prime}\right) \boldsymbol{R}_{i}{ }^{\prime}-\boldsymbol{p}_{i} \cdot \boldsymbol{R}_{i}^{\prime 2}}{\boldsymbol{R}_{i}{ }^{5}}$

$(x x)$ means that the electric vector of the applied field is parallel to $x$ and the induced field parallel to $x . x$ is perpendicular

to the film plane and $z$ is parallel to the $c$ axis .

\begin{tabular}{ccccccccc}
\hline & \multicolumn{3}{c}{$\begin{array}{c}\text { Case (1): all } p_{i} \\
\text { are the same }\end{array}$} & & \multicolumn{3}{c}{$\begin{array}{c}\text { Case (2): } p_{i} \text { is taken as } \\
\text { electron density at atom } i\end{array}$} \\
\cline { 2 - 4 } \cline { 6 - 8 } Radius of small sphere & $(x, x)$ & $(y, y)$ & $(z, z)$ & & $(x, x)$ & $(y, y)$ & $(z, z)$ \\
\hline $15 \AA$ & $0.21_{5}$ & $0.01_{5}$ & $-0.23_{0}$ & & $0.22_{1}$ & $0.00_{4}$ & $-0.22_{5}$ \\
$25 \AA$ & $0.21_{4}$ & $0.02_{1}$ & $-0.23_{6}$ & & $0.22_{0}$ & $0.01_{1}$ & $-0.23_{1}$ \\
$50 \AA$ & $0.21_{4}$ & $0.02_{2}$ & $-0.23_{6}$ & & $0.21_{9}$ & $0.01_{1}$ & $-0.23_{1}$ \\
\hline
\end{tabular}


Using eq 11, 12, 17, 18 and 19, we obtain

$$
\frac{\varepsilon(\omega)-1}{\varepsilon(\omega)+\left(\frac{4 \pi}{A}-1\right)}=\frac{A \mathrm{e}^{2}}{m} \sum_{j} N_{j} \frac{f_{j}}{\left(\omega_{0_{j}}^{2}-\omega^{2}\right)+i \gamma_{j} \omega}
$$

For the $k$-th absorption band,

$$
\operatorname{Im}\left(\frac{\mathrm{i}-\varepsilon(\omega)}{\varepsilon(\omega)+\left(\frac{4 \pi}{A}-1\right)}\right)=\frac{A \mathrm{e}^{2}}{m} \sum_{j} N_{j} \frac{f_{j} \gamma_{j} \omega}{\left(\omega_{0 j}^{2}-\omega^{2}\right)-\gamma_{j}^{2} \omega^{2}}
$$

Therefore,

$$
\left[\operatorname{Im}\left(\frac{1-\varepsilon(\omega)}{\varepsilon(\omega)+\left(\frac{4 \pi}{A}-1\right)}\right) \cdot \omega\right]_{\max }=A \sum_{j} \frac{\mathrm{e}^{2} N_{j}}{m} f_{j}
$$

Using case (2) and the radius $50 \AA$ in Table VI, we estimated $A$ to be

$$
(x x): 7.61 \quad(y y): 4.36 \quad(z z): 0.576
$$

With these figures and eq 23 , the results shown in Table VII were derived. For the second absorption band, for example, $f_{\|}=0.2_{9}, f_{\perp}=0.05_{7}$ and $f_{\|} / f_{\perp}=$ $5.0_{8}$.

The above calculations were made on the assumption that the samples consist of perfect crystals aligned exactly along the stretching direction. But, this is too simple an assumption.

As a better model, suppose that molecules are aligned in the stretching direction, with a distribution of orientation characterized by an average birefringence $\Delta n$ given by ${ }^{43}$

$$
\Delta n=\Delta n_{\max }\left(1-\frac{3}{2} \sin ^{2} \beta\right)
$$

where $\Delta n_{\max }$ is the theoretical maximum birefringence 0.23 and $\beta$ is an av rage angle between the molecular axis and the stretching direction. With

Table VII. Oscillator strengths in the absorption bands of uniaxially drawn

$(\times 5)$ PET films $47 \mu \mathrm{m}$ thick calculated by eq 23

\begin{tabular}{lllllll}
\hline & $\begin{array}{c}\text { 1st abs. } \\
\text { band }\end{array}$ & $\begin{array}{c}\text { 2nd abs. } \\
\text { band }\end{array}$ & \multicolumn{2}{c}{$\begin{array}{c}\text { 3rd abs. } \\
\text { band }\end{array}$} \\
\hline \multicolumn{1}{c}{ Polarity } & & $/ /$ & $\perp$ & $/ /$ & $\perp$ \\
\hline $\begin{array}{l}f / \text { repeat unit } \\
f \text { /electron }\end{array}$ & 0.004 & $0.2_{9}$ & $0.05_{7}$ & $0.2_{8}$ & $0.1_{8}$ \\
\hline
\end{tabular}

the measured $\Delta n$ of 0.115 for the present specimen, we obtain $\beta=35.2{ }^{\circ}$ from eq 24 . With this $\beta$ value, the $A$ factor in the second absorption band yields $A_{\|}=2.92$ and $A_{\perp}=4.30$, which lead to $f_{\|}=0.18$ and $f_{\perp}=0.05_{9}$, and $f_{\|} / f_{\perp}=3.0_{5}$.

For further improvement, the above model should be applied only to the crystalline part which constitutes $23 \%$ of the present specimen, and the rest may be considered a random phase. By so doing, the factor $A$ in the second absorption bands turns out to be $A_{\|}=3.63$ and $A_{\perp}=4.19$ which give $f_{\|}=0.14$ and $f_{\perp}=0.06$ and $f_{\|} / f_{\perp}=2.3_{3}$.

In the improved model, the oscillator strength was about $2.2-2.5$ times as small as that without the Lorentz field correction. This is a reasonable outcome in the consideration of the Lorentz field. Usually, eq 23 gives smaller values for the oscillator strength than those calculated by eq 16 for a filmlike geometry; for anthrance, ${ }^{42}$ the Lorentz field correction gives values about five times smaller.

As for the polarization ratio, the value $f_{\|} \mid f_{\perp}=$ $2.3_{3}$ in the improved model is much smaller than that $\left(f_{\|} / f_{\perp}=5.0_{8}\right)$ in the case of the perfect crystal model but rather close to that $\left(f_{\|} \mid f_{\perp}=2.1_{2}\right)$ in the case of eq 16. This is reasonable since the perfect crystal is too far away from the actual state of the PET film. Depending on the degree of crystallinity and orientation, th polarization ratio shifts from the value obtained without Lorentz field correction toward that of the perfect crystal model.

\section{Assignment of Absorption Bands}

In discussing the assignment of absorption bands, it is desirable to determine the wavefunctions, transition energies and transition moments by molecular orbital calculations. So far, quantum chemical treatment of a PET model compound has been made only by Lowe et al., ${ }^{44}$ who took terephthalaldehyde as the model and discussed the phenylenecarboxylate comformation based on calculations by extended Hückel, CNDO/INDO, MINDO/3, and $a b$ initio. Here, since the absorption spectra were the major concern, the $\pi$ electron approximation was applied to a model compound:

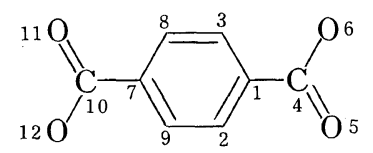

where the numbers distinguish the atoms. 


\section{OUCHI}

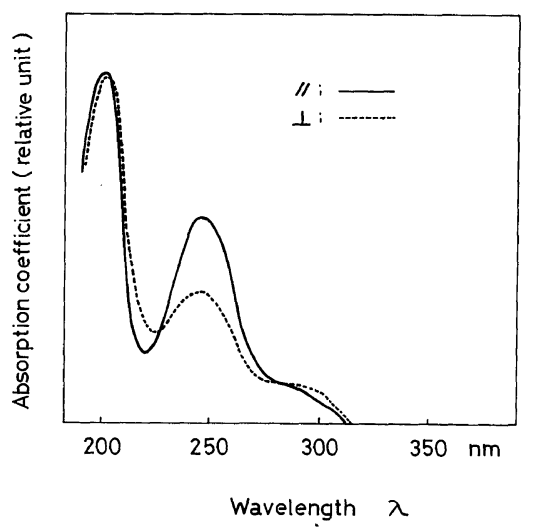

(a)

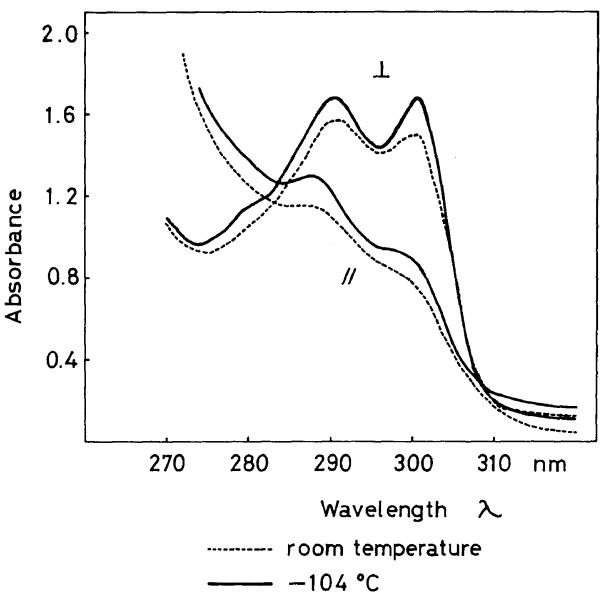

(b)

Figure 17. a. Absorption spectra of poly(trimethylene terephthalate) calculated from the reflection spectra by the Kramers-Kronig formula. b. Absorption spectra of the first absorption band of a four times uniaxially drawn poly(trimethylene terephthalate) film.

Use of this model for the $\pi$ electron approximation may be justified as a first approximation because of the close resemblance of the electronic spectra of PET to those of poly(trimethylene terephthalate) and poly(tetramethylene terephthalate).
The absorption spectra of a poly(trimethylene terephthalate) film converted from reflection spectra by the Kramers-Kronig formula, along with the directly measured spectra of the first absorption band are shown in Figure 17.

The $i$-th molecular orbital is denoted by $\psi_{i}(q)$, which is a function of the space coordinates of the $q$ th electron and is constructed by a linear combination of the atomic orbitals:

$$
\psi_{i}(q)=\sum_{\kappa=1}^{12} C_{k i} \chi_{k}(q) \quad i=1,2, \cdots, 12
$$

Here, $\chi_{k}(q)$ is the atomic orbital of the $k$-th atom and $C_{k i}$ is a coefficient. The Pariser-Parr-Pople (PPP) approach ${ }^{45-48}$ was adopted to carry out the calculation. To evaluate several types of integrals, some trial estimations were made for different approximation formulae, and the Beverage-Hinze approximation $^{49}$ was chosen for two-center Coulomb integrals:

$$
\gamma_{\mu \nu}=\frac{1}{\left[a_{\mu \nu} \exp \left(-r^{2} / 2 a_{\mu \nu}{ }^{2}\right)+r\right]}
$$

where

$$
a_{\mu \nu}=\frac{2}{(\mu \mu \mid \mu \mu)+(\nu v \mid \nu v)}
$$

with

$$
(\mu \mu \mid \mu \mu)=\int \chi_{\mu}(1) \chi_{\mu}(2) \frac{\mathrm{e}^{2}}{r_{12}} \chi_{\mu}(1) \chi_{\mu}(2) \mathrm{d} v_{12}
$$

Other integrals were computed according to Kikuchi's book. ${ }^{24}$ Several position and comformation parameters were tried, and the data given below were chosen for bond lengths and angles:

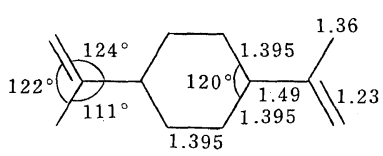

The calculated molecular orbitals and orbital energies $\left(e_{i}\right)$ in $\mathrm{eV}$ are as follows:

$$
\begin{aligned}
e_{1}=-18.239 \quad \psi_{1}= & 0.0927\left(\chi_{1}+\chi_{7}\right)+0.0449\left(\chi_{2}+\chi_{8}\right)+0.0456\left(\chi_{3}+\chi_{9}\right) \\
& +0.2876\left(\chi_{4}+\chi_{10}\right)+0.1447\left(\chi_{5}+\chi_{11}\right)+0.6194\left(\chi_{6}+\chi_{12}\right) \\
e_{2}=-18.225 \quad \psi_{2}= & -0.0813\left(\chi_{1}-\chi_{7}\right)-0.0209\left(\chi_{2}-\chi_{8}\right)-0.0201\left(\chi_{3}-\chi_{9}\right) \\
& -0.2870\left(\chi_{4}-\chi_{10}\right)-0.1444\left(\chi_{5}-\chi_{11}\right)-0.6240\left(\chi_{6}-\chi_{12}\right)
\end{aligned}
$$




$$
\begin{aligned}
e_{3}=-14.963 \quad \psi_{3}= & 0.3969\left(\chi_{1}+\chi_{7}\right)+0.3762\left(\chi_{2}+\chi_{8}\right)+0.3837\left(\chi_{3}+\chi_{9}\right) \\
& +0.1054\left(\chi_{4}+\chi_{10}\right)+0.1150\left(\chi_{5}+\chi_{11}\right)-0.1900\left(\chi_{6}-\chi_{12}\right) \\
e_{4}=-13.592 \quad \psi_{4}= & 0.2586\left(\chi_{1}-\chi_{7}\right)+0.0791\left(\chi_{2}-\chi_{8}\right)+0.1225\left(\chi_{3}-\chi_{9}\right) \\
& +0.2814\left(\chi_{4}-\chi_{10}\right)+0.5011\left(\chi_{5}-\chi_{11}\right)-0.2857\left(\chi_{6}-\chi_{12}\right) \\
e_{5}=-13.109 \quad \psi_{5}= & 0.0716\left(\chi_{1}+\chi_{7}\right)+0.1433\left(\chi_{2}+\chi_{8}\right)+0.1448\left(\chi_{3}+\chi_{9}\right) \\
& -0.2592\left(\chi_{4}+\chi_{10}\right)-0.5797\left(\chi_{5}+\chi_{11}\right)+0.2240\left(\chi_{6}+\chi_{12}\right) \\
e_{6}=-11.829 \quad \psi_{6}= & -0.1075\left(\chi_{1}-\chi_{7}\right)+0.4169\left(\chi_{2}-\chi_{8}\right)-0.5510\left(\chi_{3}-\chi_{9}\right) \\
& +0.0249\left(\chi_{4}-\chi_{10}\right)+0.1007\left(\chi_{5}-\chi_{11}\right)-0.0170\left(\chi_{6}-\chi_{12}\right) \\
e_{7}=-11.537 \quad \psi_{7}= & -0.4922\left(\chi_{1}-\chi_{7}\right)-0.3725\left(\chi_{2}-\chi_{8}\right)-0.1257\left(\chi_{3}-\chi_{9}\right) \\
& +0.0343\left(\chi_{4}-\chi_{10}\right)+0.3191\left(\chi_{5}-\chi_{11}\right)-0.009\left(\chi_{6}-\chi_{12}\right) \\
e_{8}=-1.975 \quad \psi_{8}= & 0.4274\left(\chi_{1}+\chi_{7}\right)-0.2089\left(\chi_{2}+\chi_{8}\right)-0.3065\left(\chi_{3}+\chi_{9}\right) \\
& +0.3156\left(\chi_{4}+\chi_{10}\right)-0.2598\left(\chi_{5}+\chi_{11}\right)-0.1120\left(\chi_{6}+\chi_{12}\right) \\
e_{9}=-0.866 \quad \psi_{9}= & 0.0299\left(\chi_{1}+\chi_{7}\right)-0.5273\left(\chi_{2}+\chi_{8}\right)+0.4676\left(\chi_{3}+\chi_{9}\right) \\
& +0.0362\left(\chi_{4}+\chi_{10}\right)-0.0301\left(\chi_{5}+\chi_{11}\right)-0.0105\left(\chi_{6}+\chi_{12}\right) \\
e_{10}=-0.088 \quad \psi_{10}= & -0.0616\left(\chi_{1}-\chi_{7}\right)+0.2318\left(\chi_{2}-\chi_{8}\right)+0.2180\left(\chi_{3}-\chi_{9}\right) \\
& -0.5168\left(\chi_{4}-\chi_{10}\right)+0.3214\left(\chi_{5}-\chi_{11}\right)+0.1566\left(\chi_{6}-\chi_{12}\right) \\
e_{12}=2.627 \quad \psi_{12}= & -0.4109\left(\chi_{1}-\chi_{7}\right)+0.3563\left(\chi_{2}-\chi_{8}\right)+0.3430\left(\chi_{3}-\chi_{9}\right) \\
& +0.2638\left(\chi_{4}-\chi_{10}\right)-0.1129\left(\chi_{5}-\chi_{11}\right)-0.0646\left(\chi_{6}-\chi_{12}\right) \\
\psi_{11}= & 0.3810\left(\chi_{1}+\chi_{7}\right)-0.1446\left(\chi_{2}+\chi_{8}\right)-0.1308\left(\chi_{3}+\chi_{9}\right) \\
& -0.4879\left(\chi_{4}+\chi_{10}\right)+0.2477\left(\chi_{5}+\chi_{11}\right)+0.1318\left(\chi_{6}+\chi_{12}\right) \\
e_{11} &
\end{aligned}
$$

Two uppermost filled orbitals $\psi_{6}$ and $\psi_{7}$ have orbital energies close to each other. Indeed, these degenerate if calculated by the Hückel method.

The total electron energy $E_{0}$ is expressed by ${ }^{50}$

$$
E_{0}=2 \sum_{j=1}^{6} H_{i}+\sum_{i, j=1}^{6}\left(2 J_{i j}-K_{i j}\right)
$$

where

$$
\begin{aligned}
H_{i} & =\int \psi_{i}(1) H^{\text {core }}(1) \psi_{i}(1) \mathrm{d} \tau(1) \\
J_{i j} & =\int \psi_{i}(p) \psi_{i}(p) \frac{e}{r_{p q}} \psi_{j}(q) \psi_{j}(q) \mathrm{d} \tau(p) \mathrm{d} \tau(q) \\
k_{i j} & =\int \psi_{i}(p) \psi_{j}(p) \frac{e}{r_{p q}} \psi_{i}(q) \psi_{j}(q) \mathrm{d} \tau(p) \mathrm{d} \tau(q)
\end{aligned}
$$

and $r_{p q}$ is the distance between $\pi$ electrons $p$ and $q$.

Let $\Phi_{i \rightarrow k}$ and $E_{i \rightarrow k}$ be the singlet total wavefunction and the energy corresponding to the elec- tronic configuration in which an electron is excited from the $i$-th to the $k$-th molecular orbital. Since the self-consistent field method is employed, the transition energy from the ground state is given by

$$
{ }^{1} E_{i \rightarrow k}-E_{0}=e_{k}-e_{i}-J_{i k}+2 K_{i k}
$$

The molecular symmetry of the PET model compound is $C_{2 \mathrm{~h}}$ : the character table is given in Table VIII, where the two-fold axis is taken as the $x$ axis.

The four lowest excited states $\Phi_{6 \rightarrow 8}, \Phi_{7 \rightarrow 8}, \Phi_{6 \rightarrow 9}$, and $\Phi_{7 \rightarrow 9}$ belong to the symmetry group $B_{\mathrm{u}}$, so that

Table VIII. Character table of $C_{2 h}$ groups

\begin{tabular}{llrrrr}
\hline$C_{2 h}$ & $E$ & $C_{2}(x)$ & \multicolumn{1}{c}{$i$} & \multicolumn{1}{c}{$\sigma_{h}(y, z)$} \\
\hline$A_{\mathrm{g}}$ & 1 & 1 & 1 & 1 & \\
$A_{\mathrm{u}}$ & 1 & 1 & -1 & -1 & $x$ \\
$B_{\mathrm{g}}$ & 1 & -1 & 1 & -1 & \\
$B_{\mathrm{u}}$ & 1 & -1 & -1 & 1 & $y, z$ \\
\hline
\end{tabular}


transitions from the ground state are allowed. The energies for these transitions calculated by eq 27 are $\Phi_{6 \rightarrow 8} 5.27 \mathrm{eV}, \Phi_{7 \rightarrow 8} 5.30 \mathrm{eV}, \Phi_{7 \rightarrow 9} 5.84 \mathrm{eV}$, and $\Phi_{6 \rightarrow 9}$ $6.28 \mathrm{eV}$.

To obtain more accurate results, restricted configuration interaction $(\mathrm{CI})$ calculations were per- formed; the matrix elements of the above four $B_{\mathrm{u}}$ configurations were computed for excited states. The computation was carried out using the method of Murrell et al. ${ }^{51}$ The results are given below, where $\Phi_{\mathrm{I}} \cdots \Phi_{\mathrm{IV}}$ represent the improved wavefunctions in the increasing order of energy.

$$
\begin{aligned}
& \Phi_{\mathrm{I}}=-0.3868 \Phi_{7 \rightarrow 8}+0.5003 \Phi_{7 \rightarrow 9}+0.7560 \Phi_{6 \rightarrow 8}+0.1689 \Phi_{6 \rightarrow 9}: \quad 4.66 \mathrm{eV} \\
& \Phi_{\mathrm{II}}=0.8601 \Phi_{7 \rightarrow 8}+0.2782 \Phi_{7 \rightarrow 9}+0.3194 \Phi_{6 \rightarrow 8}-0.2843 \Phi_{6 \rightarrow 9}: \quad 5.27 \mathrm{eV} \\
& \Phi_{\text {III }}=0.0232 \Phi_{7 \rightarrow 8}+0.7967 \Phi_{7 \rightarrow 9}-0.5638 \Phi_{6 \rightarrow 8}+0.2164 \Phi_{6 \rightarrow 9}: \quad 6.33 \mathrm{eV} \\
& \Phi_{\mathrm{IV}}=-0.3318 \Phi_{7 \rightarrow 8}+0.1937 \Phi_{7 \rightarrow 9}-0.0927 \Phi_{6 \rightarrow 8}-0.9186 \Phi_{6 \rightarrow 9}: \quad 6.44 \mathrm{eV}
\end{aligned}
$$

The transition moments from the ground state to the $\Phi_{i \rightarrow j}$ configuration can be calculated from

$$
\begin{aligned}
\left(m_{i j}\right)_{x} & =\int \Phi_{0} x \Phi_{i \rightarrow j} \\
& =\sqrt{2}\left(\sum_{\mu} C_{i \mu} C_{j \mu} X_{\mu}+\sum_{\mu \neq v} \sum_{i \mu} C_{j v} S_{\mu v} \frac{X_{\mu}+X_{v}}{2}\right)
\end{aligned}
$$

where $X_{\mu}$ is the coordinate of the nucleus to which the orbital $X_{\mu}$ belongs and $S_{\mu \nu}$ is the overlap integral. The $y$ and $z$ components of $m_{i j}$ are expressed in the same way. Thus, the transition moments from the ground state to the states specified by $\Phi_{\mathrm{I}} \cdots \Phi_{\mathrm{IV}}$ can be calculated from

$$
\left(M_{L}\right)_{x}=\sum_{k=1}^{4} A_{L K}\left(m_{i j}\right)_{x} \quad(L=1 \cdots \mathrm{IV})
$$

where $A_{L k}$ is the coefficient of $\Phi_{\mathrm{I}} \cdots \Phi_{\mathrm{IV}}$ described above.

Furthermore, the oscillator strength $f_{x}$ can be calculated from

$$
f_{x}=\frac{8 \pi^{2} m c}{3 h} v_{L} G\left(M_{L}\right) x^{2}
$$

where $v_{L}$ is the wavenumber for the transition from the ground state to the state corresponding to $\Phi_{L}, h$ is Planck's constant, and $G$ is a degeneracy.

The calculated results along with the observed data are illustrated in Figure 18. It can be seen that the calculated and observed absorption positions agree fairly well, although the calculated absorption maxima always appear on the higher energy side of those observed, particularly, in the first absorption band.

The calculated highest two transitions appear as the third absorption, overlapping at 6.33 and
$6.44 \mathrm{eV}$.

The polarization of the first absorption band should be noticed; the relative magnitude of the parallel component to the perpendicular one is reversed in the calculation compared with that of the experiment. When the PPP method is used, the calculated results are often affected in some degree by the given atomic coordinates of the molecules; for instance, if the configuration of the PET crystal used by Arnott et al..$^{52}$ in which the benzene ring is not of three fold symmetry and distance $C(1)-$ $C(2)=1.41 \AA, \quad C(1)-C(3)=1.34 \AA, \quad$ and $C(8)-$ $C(3)=1.41 \AA$, the calculation leads to somewhat higher $f_{\perp}$ than $f_{\|}$in the first absorption band.

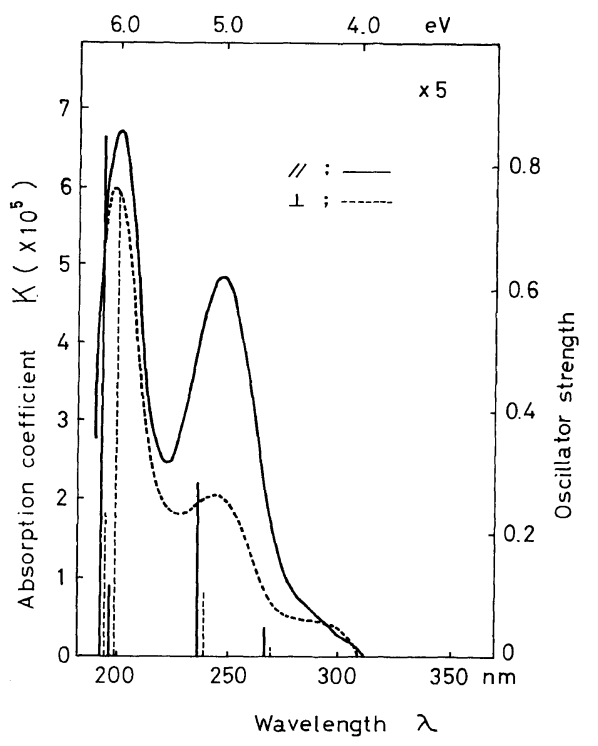

Figure 18. Schematic representation of observed and calculated electronic transitions in PET. The length of the bars represents the calculated oscillator strength. 
Two interpretations may be made for the aforementioned fact. The first is concerned with the applicability of the PPP method to the present case. In view of the considerable effect brought about by such small changes in atomic positions as mentioned above, it may not be appropriate to discuss small differences in transition energy and transition moment by the PPP method in which the parameters are originally set so as to fit the experimental data for benzene and ethylene. Therefore, when this method is applied to large molecules containing heteroatoms, no complete agreement with experiment should be expected. Even with more elaborate approximate molecular orbitals, it is not sure whether polarizability can be predicted accurately. Hashimoto et al. ${ }^{28}$ found for anisotropic electron absorption spectra of polyethylene that the calculated polarization of the two lowest transitions agrees with the experiment only when the ab initio method was used.

The second is concerned with the PET model which we used. According to the analysis of Daubeny et al. ${ }^{41}$ the carbonyl groups make an angle of $12^{\circ}$ with the benzene ring in the crystal. Within the limits of the present method, use of this angle did not particularly affect the final results, regardless of the change in symmetry from $C_{2 h}$ to $C_{i}$. However, if one considers a model in which the conformation of carbonyl groups relative to the benzene ring is cis rather than trans as

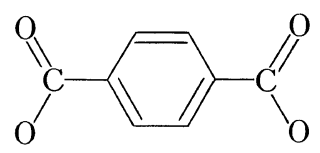

the symmetry changes to $C_{2 v}$. Then, absorption maxima remain at about the same position, but the polarization of the transition moments becomes perpendicular in the first absorption, parallel in the second absorption, and perpendicular and parallel in the two-fold third absorption band. In this case, if the transition moment is not zero along the long axis of the molecule, it is zero along the short axis and vice versa. Since this is caused by the symmetry, small displacements of atoms do not affect the results. Thus, the cis comformation explains at least qualitatively the experimental polarization data.

The established chain configuration in the crystalline portion of PET is trans since the original crystallographic analysis by Daubeny et $a l^{41}$
However, it has been shown by measurement of dipole moment that both cis and trans forms are likely to exist in the liquid state of PET.$^{53}$ Also, Saiz et al. ${ }^{54}$ from a consideration of Coulombic interactions between ester groups of DMT, concluded that the statistical weight of the cis form is 0.92 relative to the trans form. Moreover, Lowe et al. ${ }^{44}$ found from the potential energy calculation that the cis and trans forms equally exist in unoriented PET. The existence of trans conformers in crystalline and non-crystalline portions of PET films should give transition moments polarized somewhat more in the parallel direction than in perpendicular direction in the first absorption band. On the other hand, cis conformers which may exist only in non-crystalline portions in the same proportion as trans conformers should contribute only to the perpendicular components of transition moments. Thus, overall polarization should favour the perpendicular direction. If this picture is right, heat treatment of the sample will increase the parallel component of the oscillator strength in the first absorption band, since some of cis conformers are transformed into trans conformers during crystallization. This is found to be the case in Table II; the polarization ratio increased from $0.2_{8}$ for the untreated film to $0.6_{2}$ for the heat treated film in the $A^{0}$ component. The absolute difference between the data for untreated and heat treated films is very small and may be within experimental error. However, if this difference is real, then the possibility that cis conformers exist in the non-crystalline phase even in the oriented state becomes higher and explains the polarization of the first absorption band.

Acknowledgements. The author should like to express his sincere appreciation to Professor Kuniya Fukuda of Kyoto University for his continuous encouragement throughout this work. I am greatly indebted to Professor Atsuo Matsui of Konan University for his valuable suggestions and comments pertaining to the spectroscopy of the molecular crystals.

Thankful acknowledgement is also due to my colleagues at the Plastics Research Laboratories of Teijin Limited for various aids, particularly so to Mr. Sadao Kadoya for his assistance in the preparation and the measurement of ultrathin films and to Mr. Itsuo Ogiuchi for helping with the construction of a low temperature Dewar and reflection 
attachment.

\section{REFERENCES}

1. T. B. Marcotte, D. Cambell, J. A. Cleveland, and D. T. Turner, J. Polym. Sci., A-1, 5, 481 (1967).

2. Y. Takai, T. Osawa, K. C. Kao, T. Mizutani, and M. Ieda, Jpn. J. Appl. Phys., 14, 473 (1975).

3. Y. Takai, T. Osawa, T. Mizutani, and M. Ieda, $J$. Polym. Sci., Polym. Phys. Ed., 15, 945 (1977).

4. D. H. Phillips and J. C. Schug, J. Chem. Phys., 50, 3297 (1969).

5. R. G. Merrill and C. W. Roberts, J. Appl. Polym. Sci., 21, 2745 (1977).

6. Y. Takai, T. Mizutani, and M. Ieda, Jpn. J. Appl. Phys., 17, 651 (1978)

7. N. S. Allen and J. F. McKellar, Makromol. Chem., 179, 523 (1978).

8. P-S. R. Cheung, C. W. Roberts, and K. B. Wagener, J. Appl. Polym. Sci., 24, 1809 (1979).

9. J. A. Dellinger and C. W. Roberts, J. Appl. Polym. Sci., 26, 321 (1981).

10. I. Ouchi, Rep. Prog. Polym. Phys. Jpn., 20, 435 (1977).

11. M. R. Padhye and P. S. Tamhane, Angew. Makromol. Chem., 69, 33 (1978).

12. T. S. Robinson, Proc. Phys. Soc., B65, 910 (1952).

13. T. S. Robinson and W. C. Price, Proc. Phys. Soc., 66, 969 (1953).

14. J. G. Carter, T. M. Gelinek, R. N. Hamm, and R. D. Birkoff, J. Chem. Phys., 44, 2266 (1966).

15. W. Möller, H. P. Geserich, and L. Pintschovices, Solid State Commun., 18, 791 (1976).

16. A. A. Bright, M. J. Cohen, A. F. Garito, A. J. Heeger, C. M. Mikulshi, and A. G. MacDiarmid, Appl. Phys. Lett., 26, 612 (1975).

17. I. Ouchi, Rep. Prog. Polym. Phys. Jpn., 22, 437 (1979).

18. E. T. Arakawa, M. W. Williams, J. C. Ashley, and L. R. Painter, J. Appl. Phys., 52, 3579 (1981).

19. R. G. Parr, "The Quantum Theory of Molecular Electronic Structure," W. A. Benjamin, Reading, Massachusetts, 1963.

20. S. Hashimoto and H. Inokuchi, Polym. J., 8, 467 (1976).

21. B. Rossi, "Optics," Addison-Wesley, Reading, Mass., 1956.

22. S. Shionoya, T. Karatsu, and H. Era, "Optical Measurements, Kōgakuteki Sokutei,” by S. Iida, K. Ohno, T. Kanzaki, H. Kumagaya, and S. Sawada, Ed., Asakura Shoten, Tokyo, 1967, p 414.

23. R. Kato, J. Phys. Soc. Jpn., 16, 2525 (1961).

24. O. Kikuchi, "Molecular Orbital Methods-Applications of Computers, Bunshi Kidōhō," Kodansha, Tokyo, 1971.

25. H. Baba, "Measurements of Electronic Spectra," in "Jikken Kagaku Koza," Vol. 11, The Chemical
Society of Japan, Maruzen, Tokyo, 1965, p 23.

26. S. Nagakura, J. Tanaka, J. Nakamura, H. Hosoya, I. Hanasaki, and S. Iwata, "Theory of Electronic Spectra of Molecules," in "Jikken Kagaku Koza," Vol. 11, The Chemical Society of Japan, Maruzen, Tokyo, 1965, p 278

27. R. N. Nurmukhametov and G. I. Grishna, Opt. Spectrosc., 24, 107 (1968).

28. S. Hashimoto, K. Seki, N. Sato, and H. Inokuchi, $J$. Chem. Phys., 76, 163 (1982).

29. I. M. Ward and M. A. Wilding, Polymer, 18, 327 (1977).

30. S. K. Bahl, D. D. Cornell, F. J. Boerio, and G. E. McGraw, J. Polym. Sci., Polym. Lett. Ed., 12, 13 (1974).

31. F. J. Boerio, S. K. Bahl, and G. E. McGraw, J. Polym. Sci., Polym. Phys. Ed., 14, 1029 (1976).

32. J. C. Baum and D. S. McClure, J. Am. Chem. Soc., 101, 2335 (1979).

33. M. Born and E. Wolf, "Principles of Optics," Pergamon Press, London, 1964, p 617.

34. K. Kudo, "Hikari Bussei no Kiso, (Fundamentals of Optical Properties of Solids)," Ohm Sha, Tokyo, 1977, p 108.

35. D. M. Roessler, Br. J. Appl. Phys., 16, 1119 (1965).

36. C. Kittel, "Introduction to Solid State Physics," Wiley-Maruzen, New York and Tokyo, 1956, p 166.

37. M. Kotani, E. Ishiguro, K. Takayanagi, K. Ohno, and K. Itoh, "Genshi Bunshi no Ryoshi Rikigaku (Quantum Mechanics of Atoms and Molecules)," in Iwanami Koza-Gendai Butsurigaku (Series on Modern Physics) II.A, Iwanami, Tokyo, 1955, p 238.

38. M. Altarelli, D. L. Dexter, and H. M. Nussenzveig, Phys. Rev., B, 6, 4502 (1972).

39. J. Yamashita, "Yudentai (Dielectrics)," in Iwanami Koza-Gendai Butsurigaku (Series on Modern Physics), II.G, Iwanami, Tokyo, 1955, p 7.

40. R. Silbey, J. Jortner, and S. A. Rice, J. Chem. Phys., 42, 1515 (1965).

41. R. P. Daubeny, C. W. Bunn, and C. J. Brown, Proc. R. Soc. London, Ser. A, 226, 531 (1954).

42. A. Matsui and Y. Ishii, J. Phys. Soc. Jpn., 23, 581 (1967).

43. M. Kashiwagi, A. Cunningham, A. J. Manuel, and I. M. Ward, Polymer, 14, 111 (1973).

44. L. M. Lowe and E. Sacher, J. Macromol. Sci., Phys. B15, 619 (1978).

45. R. Pariser and R. G. Parr, J. Chem. Phys., 21, 767 (1953).

46. R. G. Parr and R. Pariser, J. Chem. Phys., 23, 711 (1955).

47. J. A. Pople, Trans. Faraday Soc., 49, 1375 (1953).

48. J. A. Pople, Proc. R. Soc. London, 98, 81 (1955).

49. D. L. Beveridge and J. Hinze, J. Am. Chem. Soc., 93, 3107 (1971).

50. T. Yonezawa, C. Nagata, H. Kato, A. Imamura, and K. Morokuma, "Rhyoshi Kagaku Numon 


\section{Absorption and Reflection Spectra of PET}

(Introduction to Quantum Chemistry)," Kagaku Dojin, Kyoto, 1969, p 380.

51. J. N. Murrell and K. L. McEwen, J. Chem. Phys., 25, 1143 (1956).

52. S. Arnott and A. J. Wonacott, Polymer, 7, 157
(1966).

53. E. N. Gur'ganova and N. I. Grishki, J. Struct. Chem. (USSR) (Eng. Trans.), 4, 339 (1963).

54. E. Saiz, J. P. Hummel, and P. J. Flory, J. Phys. Chem., 85, 3211 (1981). 